\title{
Self-rated joint hypermobility: the five-part questionnaire evaluated in a Swedish non- clinical adult population
}

\author{
Martin Glans ${ }^{1 *}$ (D), Mats B. Humble ${ }^{1,2}$, Marie Elwin ${ }^{1}$ and Susanne Bejerot ${ }^{1,2,3}$
}

\begin{abstract}
Background: The conventional way to identify generalised joint hypermobility is by a physical examination according to the Beighton Score. However, a physical examination is time-consuming in clinical practise and may be unfeasible in population-based studies. The self-assessment five-part questionnaire on hypermobility (5PQ) offers a more practicable way to identify GJH. The aim of this study was to test validity and reliability of the five-part questionnaire on hypermobility (5PQ) translated into Swedish on a non-clinical adult population.

Methods: A structured procedure was used for the translation of the 5PQ into Swedish. The Beighton Score was used as reference standard for generalised joint hypermobility. Test-retest reliability was tested in a separate group who filled in the questionnaire twice with a ten-week interval. Participants consisted of a convenience sample recruited in Stockholm, Sweden (2017).
\end{abstract}

Results: A total of 328 participants were included in the study, 297 participants in the validity group and 31 participants in the reliability group. When evaluated against a present Beighton Score with an age-dependent cutoff, the Swedish $5 \mathrm{PQ}$ attained a sensitivity of $91 \%$, a specificity of $75 \%$ and an area under the curve of 0.87 . The Swedish 5PQ showed substantial to almost perfect test-retest reliability.

Conclusions: The Swedish $5 \mathrm{PQ}$ is a valid and reliable instrument to screen for or to identify generalised joint hypermobility.

Keywords: Hypermobility, Joint instability, Surveys and questionnaires, Validation, Translation, Diagnostic self evaluation

\section{Background}

Generalised joint hypermobility $(\mathrm{GJH})$ is a term used to describe the ability to extend synovial joints beyond their normal limits [1]. GJH can be asymptomatic or symptomatic. When symptoms are present, it might be part of generalised hypermobility spectrum disorder or a heritable disorder of connective tissue, such as EhlersDanlos syndrome [1]. GJH seems to occur in $10-20 \%$ in the general population although its presence is influenced by age, gender and ethnicity [2]. The conventional way to identify GJH is by a physical examination according to the Beighton Score (BS) [3]. However, a physical

\footnotetext{
* Correspondence: glansmartin@gmail.com

${ }^{1}$ Faculty of Medicine and Health, University Health Care Research Centre,

Örebro University, Örebro, Sweden

Full list of author information is available at the end of the article
}

examination is time consuming in clinical practise and may be unfeasible in population-based studies. The selfassessment five-part questionnaire on hypermobility (5PQ) [4] offers a more practicable way to identify GJH. The 5PQ was initially developed in English to identify people with symptomatic hypermobility however, it has also been shown to be helpful for identifying asymptomatic GJH in population based studies [5]. To our knowledge the only published translation is into BrazilianPortuguese [6].

\section{The aim of this study}

The aim of this study was to test validity and reliability of the 5PQ translated into Swedish on a normal adult population. 


\section{Methods}

\section{Study population}

A total of 297 participants were recruited for the validity test. This was based on a sample size calculation [7] with an expected sensitivity and specificity of $80 \%$ respectively; an expected prevalence of GJH of $20 \%$ and a desired absolute precision of $10 \%$. Participants consisted of a convenience sample recruited in Stockholm, Sweden (2017) from various settings: a university campus $(n=168)$, a community health centre $(n=112)$ and the marketing department at the national public TV broadcaster $(n=17)$. An additional sample of 31 university students was recruited for reliability tests.

Inclusion criteria were age 18-65 years and a full understanding of the Swedish language. Exclusion criteria were a physical condition hampering the BS assessment or having any missing data on the Swedish 5PQ. Participants were informed that the aim was to collect data on joint mobility in an adult population.

\section{Measures \\ The reference standard -the Beighton score (BS)}

The BS measures hypermobility at nine joints. Traditionally, hypermobility was suggested if $\geq 4$ oof the 9 joints were hypermobile in an adult [2]. According to recent recommendations [8], for the diagnosis of hypermobile Ehlers-Danlos syndrome, the BS cut-off for GJH in pubertal men and women up to 50 years is $\geq 5 / 9$ while the cut-off of $\geq 4 / 9$ is used for adults 50 years and above. Furthermore, the inclusion of historical information on hypermobility by the means of the $5 \mathrm{PQ}$ is suggested for individuals with a physical condition hampering the BS assessment. If the $\mathrm{BS}$ is 1 point below the cut-off and the $5 \mathrm{PQ}$ is positive, then a diagnosis of GJH still can be made. Since the goal of our study was to validate the $5 \mathrm{PQ}$ we did not want to include it in the reference test.

\section{The index test -the five-part questionnaire on hypermobility (5PQ)}

The 5PQ consists of five items. Each endorsement yields one point and two or more points suggests GJH [4]

Table 1 The Five-Part Questionnaire (5PQ) [4] for defining Generalised Joint Hypermobility

1. Can you now (or could you ever) place your hands flat on the floor without bending your knees?

2. Can you now (or could you ever) bend your thumb to touch your forearm?

3. As a child did you amuse your friends by contorting your body into strange shapes OR could you do the splits?

4. As a child or teenager did your shoulder or kneecap dislocate on more than one occasion?

5. Do you consider yourself double-jointed?

Endorsement of two or more questions suggests generalised joint hypermobility.
(Table 1). The 5PQ was developed using the BS as a reference standard test and sensitivity and specificity have been reported between $71-84 \%$ and $77-89 \%$ respectively $[4,6]$. The Brazilian-Portuguese validation of the 5PQ applied a present BS of $\geq 4 / 9$ as a cut-off for GJH [6]. The original 5PQ authors did not clearly specify whether historical hypermobility was included in their validating BS tests [4].

\section{Procedures \\ Translation of the questionnaire into Swedish}

The original authors granted permission to translate the 5PQ. Forward translation was done by a health professional, familiar with terminology of the area, native in Swedish and with a good command of English (the first author, MG). A panel including the last author SB (medical doctor, with a wide experience in validating rating scales); the first author MG (medical doctor) and a layman native in English and fluent in Swedish discussed the preliminary Swedish version with regard to language and cultural applicability before a consensual version was achieved. Two professional translators, native in English and unaware of the original version, back-translated the preliminary adaptation. The back-translated versions were compared to the original questionnaire whereby a final version was agreed upon. The final version was communicated to the original authors. The translation process is shown in Supplementary Table S1, Additional file 1. The Swedish 5PQ Screen print-out version with instructions to the clinician is available as supportive information in Additional file 3 .

When the original version of the $5 \mathrm{PQ}$ is compared to our back-translated versions (Supplementary Table S1, Additional file 1), we consider item 3 "As a child did you amuse your friends by contorting your body into strange shapes OR could you do the splits?" and item 5 "Do you consider yourself double-jointed?" to differ the most from the original version. Regarding item 3, the words "amuse", "contort" and "shapes" were back-translated to "entertain", "twist" and "positions", which we consider being correct synonyms. Regarding item 5, "double-jointed" was backtranslated to "clearly hypermobile in your joints" and "to have joint hypermobility". There is no Swedish expression equivalent to "double-jointed", and the Swedish phrasing was considered as informal. We believe that the differences in wording were not such as to affect the overall understanding or interpretability of the Swedish version of the 5PQ.

\section{Data collection}

Participants completed a survey including the Swedish 5PQ and demographic data, followed by a clinical examination with the BS. A goniometer was used to assess the fifth finger, elbow and knee (standing). The examiner was a trained physician, blinded to the results of the 
Swedish 5PQ (the first author, MG). The subjects did not warm up prior to the examination. Any physical condition hampering the examination was recorded. For reliability testing, the Swedish 5PQ was administered and re-administered after 10 weeks to a subgroup of 31 subjects. The reliability group did not undergo a physical examination.

\section{Statistics}

Statistical analyses were primarily conducted in IBM SPSS statistics version 23. MedCalc version 18.5 was used to calculate $95 \%$ confidence intervals on sensitivity, specificity, positive predictive value (PPV) and negative predictive value (NPV).

\section{Validity}

The validity of the Swedish 5PQ was tested against two different criteria of the reference standard test: 1) a present BS with an age-dependent cut-off of $\geq 5 / 9$ for individuals $18-50$ years and $\geq 4 / 9$ for individuals $>50$ years, according to the updated recommendations and 2) a present BS with the traditional cut-off of $\geq 4 / 9$. Post hoc analyses were performed on additional cut-off scores on the reference standard test. Non-parametric assumptions about the distribution were adopted in the construction of the receiver operator characteristic (ROC) curves. Area under the curve (AUC) was interpreted as no discrimination (0-0.5), poor (0.5-0.7), fair $(0.7-0.8)$, good $(0.8-0.9)$ or excellent $(0.9-1.0)$ accuracy [9]. There are no agreed standards by which to judge sensitivity and specificity, rather the optimal balance between sensitivity and specificity depends on the purpose of the test. Generally, a screening test should prioritise a high sensitivity, while a follow-up confirmatory test should be highly specific [10]. Predictive values are, on the other hand, not only dependent on sensitivity and specificity but also on the prevalence of the disease in the studied population. Accordingly, in populations with a high prevalence of the disease, the PPV will be higher than in populations or settings where the disease is rare [10].

\section{Test-retest reliability}

Test-retest reliability for each item and for the totalscore of the Swedish 5PQ was examined by Cohen's unweighted kappa and intra-class correlation coefficient (ICC), respectively. Cohen's kappa was interpreted as slight (0-0.2), fair (0.2-0.4), moderate (0.4-0.6), substantial (0.6-0.8) and almost perfect $(0.8-1.0)$ agreement [10]. ICC estimates were calculated based on a singlemeasurement, absolute agreement, two-way mixed-effect model. ICC was interpreted as poor $(0-0.5)$, moderate (0.5-0.75), good (0.75-0.9) or excellent (0.9-1.0), [11].

\section{Item specific analyses}

The distribution of responses for each item on the $5 \mathrm{PQ}$ was determined. Odds ratios (OR) for GJH for each item were calculated by $2 \times 2$ crosstabulation tables and analysed with Pearson's Chi-square test. Fisher's exact test was used when expected value of a cell was less than 5 . $P$ values reported are 2 -sided.

\section{Results}

\section{Characteristics of the study population}

A total of 328 subjects were included in the study: 297 subjects in the validity group and 31 subjects in the reliability group. In the validity group, 13 subjects were excluded due to a physical condition that hindered physical examination (Fig. 1). Out of the remaining 284 subjects, $60 \%(n=170)$ were women and $40 \%(n=114)$ were men. The mean $( \pm \mathrm{SD})$ age was $31.5(12.2)$ years. $25 \%(n=70)$ of the subjects had either one or both parents born outside of the Nordic countries: (fathers/ mothers) respectively; Europe (18/15), Asia (27/30), North America (2/2), South America (5/7) and Africa (6/4), and 4 subjects had missing data on this item. $58 \%$ of the participants had on-going university studies, $1 \%$ had completed elementary school or less, $20 \%$ had completed senior high school, $7 \%$ had completed less than 3 years of university or college studies and $14 \%$ had completed 3 years or more of university or college studies. In the reliability group $68 \%(n=21)$ were women, $32 \%(n=$ 10) were men and the mean $( \pm \mathrm{SD})$ age was $24.8(3.0)$ years. There was no missing data on the $5 \mathrm{PQ}$ in any of the groups. As expected, GJH was more frequent amongst women than among men (Table 2).

\section{Statistics \\ Validity}

When evaluated against the updated criteria of the reference test (a present BS with an age-dependent cut-off of $\geq 5 / 9$ for individuals $18-50$ years and $\geq 4 / 9$ for individuals $>50$ years), the Swedish 5PQ attained a sensitivity of $91 \%$ (95\% CI $72,99 \%$ ) a specificity of $75 \%$ (95\% CI 69 , $80 \%$ ) and an area under the curve of 0.87 (95\% CI 0.79 , 0.95). When evaluated against the traditional recommendations of the reference test (a BS according to present hypermobility, cut-off $\geq 4 / 9$ ), the Swedish $5 \mathrm{PQ}$ attained a sensitivity of $72 \%$ ( $95 \%$ CI $57,84 \%$ ), a specificity of $78 \%$ (95\% CI 72, 83\%) and an area under the curve of 0.77 (95\% CI $0.68,0.85)$. A more detailed presentation is shown in Table 3. Post hoc analyses on additional cutoff scores on the reference test are presented in Supplementary Table S2, Additional file 2.

\section{Test-retest reliability}

All aspects of the 5PQ showed substantial to almost perfect agreement. In the item-to-item comparison, kappa 


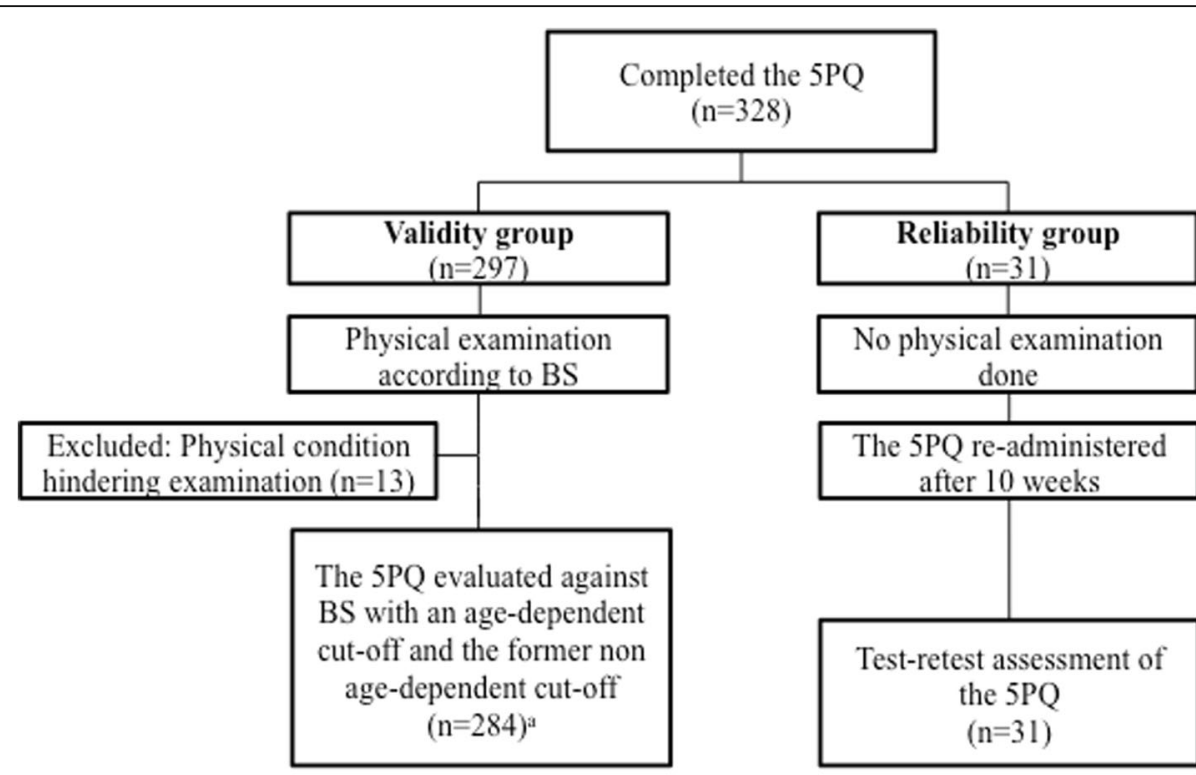

5PQ: Five-part questionnaire on hypermobility BS: Beighton score

Fig. 1 Flow of participants through the study. ${ }^{a}$ The Swedish $5 P Q$ was tested against two different criteria of the reference standard test: 1) a present Beighton Score with an age-dependent cut-off of $\geq 5 / 9$ for individuals $18-50$ years and $\geq 4 / 9$ for individuals $>50$ years and 2) a present BS with the traditional cut-off of $\geq 4 / 9$

Table 2 The distribution of hypermobility according to the applied assessment method

\begin{tabular}{lll}
\hline Assessment method & \multicolumn{3}{l}{ Hypermobile } & \\
\cline { 2 - 3 } & Yes & No \\
\hline
\end{tabular}

5PQ (Reliability group, first assessment)

$\begin{array}{lll}\text { Women: } \mathrm{n}(\%) & 6(28.6) & 15(71.4) \\ \text { Men: } \mathrm{n}(\%) & 3(30.0) & 7(70.0) \\ \text { 5PQ (Validity group) } & & \\ \text { Women: } \mathrm{n}(\%) & 65(38.2) & 105(61.8) \\ \text { Men: } \mathrm{n}(\%) & 22(19.3) & 92(80.7)\end{array}$

BS according to present hypermobility with an age-dependent cut-off score

$\begin{array}{lll}\text { Women: } \mathrm{n}(\%) & 20(11.8) & 150(88.2) \\ \text { Men: } \mathrm{n}(\%) & 3(2.6) & 11(97.4)\end{array}$

BS according to present hypermobility $\geq 4 / 9^{a}$
Women: $\mathrm{n}(\%)$
$42(24.7)$
$128(75.3)$
Men: $\mathrm{n}(\%)$
$5(4.4)$
109 (95.6)

$5 P Q$ The five-part questionnaire on hypermobility $B S$ Beighton Score

${ }^{2}$ Two different criteria of the reference standard test were used to identify generalised joint hypermobility: 1) a present Beighton Score with an age-dependent cut-off of $\geq 5 / 9$ for individuals $18-50$ years and $\geq 4 / 9$ for individuals $>50$ years and 2) a present BS with the traditional cut-off of $\geq 4 / 9$ values for each item were respectively: item $1(0.87)$, item 2 (0.83), item 3 (1.00), item $4(-)$ and item 5 (0.71). Due to absent variance in item 4, kappa measurement of agreement was not applicable. ICC for the total-score comparison was $0.92(95 \%$ CI $0.85,0.96)$.

\section{Item specific analyses}

The distribution of responses for each item of the $5 \mathrm{PQ}$, including the odds ratio (OR) for $\mathrm{GJH}$, is presented in Table 4. Item 4 "As a child or teenager did your shoulder or kneecap dislocate on more than one occasion?" fell out as non-significant to predict GJH (OR 1.56, $P=0.64$ when tested against a present BS with an age-dependent cut-off and OR 1.09, $P=1.00$ when tested against a BS according to present hypermobility $\geq 4 / 9$ ).

\section{Discussion}

In this study we tested validity and reliability of the 5PQ translated into Swedish on a normal adult population. We did not differentiate between asymptomatic and symptomatic GJH. When evaluated against the updated criteria of the reference standard test (a present Beighton Score with an age-dependent cut-off of $\geq 5 / 9$ for individuals $18-50$ years and $\geq 4 / 9$ for individuals $>$ 50 years) the Swedish $5 \mathrm{PQ}$ attained a sensitivity of $91 \%$, 
Table 3 Evaluation of validity of the Swedish $5 \mathrm{PQ}$

\begin{tabular}{|c|c|c|c|c|}
\hline 5PQ score & Sensitivity (\%) $(95 \% \mathrm{Cl})$ & Specificity (\%) (95\% Cl) & PPV (\%) (95\% Cl) & NPV (\%) $(95 \% \mathrm{Cl})$ \\
\hline \multicolumn{5}{|c|}{ BS according to present hypermobility with an age-dependent cut-off score ${ }^{a}$} \\
\hline$=0 / 5$ & $100.00(85.18,100.00)$ & $0.00(0.00,1.40)$ & $8.10(8.10,8.10)$ & - \\
\hline$\geq 1 / 5$ & $95.65(78.05,99.89)$ & $34.10(28.37,40.20)$ & $11.34(10.16,12.64)$ & $98.89(92.85,99.84)$ \\
\hline$\geq 2 / 5^{b}$ & $91.30(71.96,98.93)$ & $74.71(68.98,79.87)$ & $24.14(19.96,28.88)$ & $98.98(96.28,99.73)$ \\
\hline$\geq 3 / 5$ & $56.52(34.49,76.81)$ & $91.57(87.52,94.64)$ & $37.14(25.67,50.27)$ & $95.98(93.74,97.45)$ \\
\hline$\geq 4 / 5$ & $30.43(13.21,52.92)$ & $97.32(94.55,98.92)$ & $50.00(27.75,72.25)$ & $94.07(92.37,95.42)$ \\
\hline$=5 / 5$ & $0.00(0.00,14.82)$ & $99.62(97.88,99.99)$ & 0 & $91.87(91.82,91.93)$ \\
\hline \multicolumn{5}{|c|}{ BS according to present hypermobility $\geq 4 / 9^{a}$} \\
\hline$=0 / 5$ & $100.00(92.45,100.00)$ & $0.00(0.00,1.54)$ & $16.55(16.55,16.55)$ & - \\
\hline$\geq 1 / 5$ & $85.11(71.69,93.80)$ & $35.02(28.96,41.46)$ & $20.62(18.24,23.21)$ & $92.22(85.42,96.00)$ \\
\hline$\geq 2 / 5^{b}$ & $72.34(57.36,84.38)$ & $77.64(71.79,82.78)$ & $39.08(32.31,46.30)$ & $93.40(89.87,95.76)$ \\
\hline$\geq 3 / 5$ & $42.55(28.26,57.82)$ & $93.67(89.78,96.41)$ & $57.14(42.45,70.67)$ & $89.16(86.51,91.33)$ \\
\hline$\geq 4 / 5$ & $17.02(7.65,30.81)$ & $97.47(94.57,99.07)$ & $57.14(32.66,78.56)$ & $85.56(83.86,87.10)$ \\
\hline$=5 / 5$ & $0.00(0.00,7.55)$ & $99.58(97.67,99.99)$ & 0 & $83.39(83.28,83.51)$ \\
\hline
\end{tabular}

$5 P Q$ The Five-part questionnaire on hypermobility

$B S$ Beighton Score

PPV Positive predictive value

NPV Negative predictive value

${ }^{\text {a }}$ The Swedish $5 P Q$ was tested against two different criteria of the reference standard test: 1) a present Beighton Score with an age-dependent cut-off of $\geq 5 / 9$ for individuals $18-50$ years and $\geq 4 / 9$ for individuals $>50$ years and 2 ) a present BS with the traditional cut-off of $\geq 4 / 9$

${ }^{b}$ The recommended cut-off score for generalised joint hypermobility on the $5 \mathrm{PQ}[4]$

Table 4 The distribution of responses to each item on the $5 \mathrm{PQ}$ and the OR for GJH

\begin{tabular}{|c|c|c|c|c|c|c|}
\hline \multirow{3}{*}{$\begin{array}{l}5 \mathrm{PQ} \\
\text { item } \\
\text { number }\end{array}$} & \multicolumn{2}{|c|}{ Hypermobile } & \multicolumn{2}{|c|}{ Non-hypermobile } & \multirow{3}{*}{$\begin{array}{l}\text { OR for GJH (95\% } \\
\text { Cl) }\end{array}$} & \multirow[t]{3}{*}{$P$ value ${ }^{a}$} \\
\hline & \multicolumn{4}{|c|}{ Distribution of answers on the $5 \mathrm{PQ}$} & & \\
\hline & $\overline{Y e s}$ & No & Yes & No & & \\
\hline \multicolumn{7}{|c|}{ BS according to present hypermobility with an age-dependent cut-off score ${ }^{b}$} \\
\hline 1 & 20 & 3 & 133 & 128 & $6.42(1.86,22.12)$ & 0.001 \\
\hline 2 & 16 & 7 & 59 & 202 & $7.83(3.07,19.92)$ & $<0.001$ \\
\hline 3 & 11 & 12 & 45 & 216 & $4.40(1.83,10.60)$ & 0.001 \\
\hline 4 & 2 & 21 & 15 & 246 & $1.56(0.34,7.30)$ & 0.637 \\
\hline 5 & 14 & 9 & 16 & 245 & $23.82(8.96,63.36)$ & $<0.001$ \\
\hline \multicolumn{7}{|c|}{ BS according to present hypermobility $\geq 4 / 9^{b}$} \\
\hline 1 & 35 & 12 & 118 & 119 & $2.94(1.46,5.94)$ & 0.002 \\
\hline 2 & 29 & 18 & 46 & 191 & $6.69(3.42,13.08)$ & $<0.001$ \\
\hline 3 & 18 & 29 & 38 & 199 & $3.25(1.64,6.43)$ & $<0.001$ \\
\hline 4 & 3 & 44 & 14 & 223 & $1.09(0.30,3.94)$ & 1.000 \\
\hline 5 & 17 & 30 & 13 & 224 & $9.76(4.32,22.09)$ & $<0.001$ \\
\hline
\end{tabular}

$5 P Q$ The five-part questionnaire on hypermobility

$B S$ Beighton Score

OR Odds ratio

GJH Generalised joint hypermobility

a Pearson's chi-square test or Fisher's exact test when appropriate (2-sided)

${ }^{\mathrm{b}}$ The Swedish $5 \mathrm{PQ}$ was tested against two different criteria of the reference standard test: 1) a present Beighton Score with an age-dependent cut-off of $\geq 5 / 9$ for

individuals $18-50$ years and $\geq 4 / 9$ for individuals $>50$ years, adapted to the updated recommendations [8] and 2) a present BS with the traditional cut-off of $\geq 4 / 9$ 
a specificity of $75 \%$ and an AUC of 0.87 to identify $\mathrm{GJH}$. All aspects of the 5PQ showed substantial to almost perfect test-retest reliability.

\section{Item specific analyses}

The item 4 "As a child or teenager did your shoulder or kneecap dislocate on more than one occasion?" in the $5 \mathrm{PQ}$ did not predict $\mathrm{GJH}$ in our sample. It seems clear from Hakim and Grahame's original 5PQ study [4] that item 4 was relevant mainly among hypermobile individuals with musculoskeletal symptoms, whereas our study assessed a non-clinical population, in which dislocation of shoulder or kneecap is rare.

\section{What is the intended use and clinical role of the $5 \mathrm{PQ}$ ?}

The 5PQ was initially developed as a screening tool in patients at high risk [4] but has also been shown to be helpful for identifying $\mathrm{GJH}$ in population based studies [5].

The Swedish 5PQ attained similar sensitivity and specificity to previous reports $[4,6]$. However, the PPV was rather low. Presumably, this is a consequence of evaluating a Swedish non-clinical population and our use of the stricter criteria of the reference standard test. Together this results in a low prevalence of GJH. Accordingly, the PPV in our study would have been similar to the PPV reported in the Brazilian study [6] if the prevalence of GJH in our study group had been similar. Unfortunately these estimates were not reported in the original study [4].

In summary, our findings suggest that the $5 \mathrm{PQ}$ can be applied to identify GJH in studies where a physical examination is impractical, e.g. population studies. However, one needs to consider the likely prevalence of GJH in the targeted population. On the other hand, the $5 \mathrm{PQ}$ might actually be superior at identifying $\mathrm{GJH}$ than the $\mathrm{BS}$, since the $5 \mathrm{PQ}$ inquires for a subjective general view on hypermobility, rather than focusing exclusively on 5 joints.

\section{The BS as reference standard}

The human body has more than 230 movable or semimovable joints [12] and the BS is limited to a few joints only. Hence, the BS will disregard hypermobility in other joints. We chose, however, to limit our study to the BS as a reference standard since this was used in the original development of the 5PQ [4].

\section{Prevalence rates of GJH in our study}

Comparisons of prevalence must take age, gender, ethnicity, targeted population and the criterion used to define it into account, since the occurrence of GJH varies widely depending on these factors.
Considering the characteristics of the study population and the applied assessment method the Swedish 5PQ yielded a prevalence rate of GJH reasonably in accordance with previous reports. In our study the 5PQ assigned $38.2 \%$ of the females and $19.3 \%$ of the males as $\mathrm{GJH}$. This is similar to the prevalence rates reported in a large $(n=1039)$ Swedish study [13] also using the Swedish 5PQ as an assessment method for GJH. One study of female twins in the UK [5] reported the prevalence rate of $\mathrm{GJH}$ to be around $20 \%$, although participants aged 20-30 years (similar age to our participants) had a prevalence rate $(34 \%)$, closer to our findings. In the Brazilian study [6] the $5 \mathrm{PQ}$ rated $43.5 \%$ of the females and $28.4 \%$ of the males as GJH. Their slightly higher prevalence of GJH may be related to ethnic origin.

However, when using the $\mathrm{BS}$ as an assessment method to identify $\mathrm{GJH}$, there was a low prevalence amongst males in our study. Nevertheless, one study of American students attending a military academy reported even lower prevalence rates than we did [14]. Considerably higher rates of $\mathrm{GJH}$ were reported in another cohort of college students in the United States [15]; $13.7 \%$ of the males and $36.7 \%$ of the females were hypermobile. In their study sample, $81.3 \%$ were of North American Caucasian origin and the mean age was 20 years, hence their study is rather comparable to ours even though the age difference possibly could explain some of the difference. We have not found a comparable study describing the prevalence of GJH in Sweden. However, by interpreting the graphs presented in a Swedish study [16] that also used the BS but with a slightly different scoring system, their reported prevalence of GJH seem rather similar to ours. Assuming that $\geq 3 / 5$ hypermobility features equals the cut-off of $\geq 5 / 9$ that we applied, they classified approximately 7\% of the males as GJH compared to our finding of approximately $4 \%$. Possibly, divergent findings may also be related to the examiner [17].

\section{Translation considerations}

Item 1 "Can you now (or could you ever) place your hands flat on the floor without bending your knees?" and item 2 "Can you now (or could you ever) bend your thumb to touch your forearm?" in the 5PQ are included in the BS. In our sample, $1.5 \%$ (item 1) and 23\% (item 2 ) of the subjects who had responded negatively to the question in the $5 \mathrm{PQ}$ nevertheless had a present ability to do the manoeuvre in the BS assessment. The Brazilian study [6] provided an illustration of item 2 in their questionnaire. Nonetheless, $24 \%$ of the Brazilian participants who had responded negatively in the $5 \mathrm{PQ}$ had at least one positive score on the manoeuvre in the BS assessment. Seemingly, an illustration is insufficient to 
attain satisfactory understanding. If a revised version of the $5 \mathrm{PQ}$ is to be developed we propose the phrasing "Can you now (or could you ever) bend either thumb to touch your forearm? Please try on both sides following the illustration".

Furthermore, item 4 "As a child or teenager did your shoulder or kneecap dislocate on more than one occasion?", might be easily misinterpreted. During our study we gradually realised that subjects failed to acknowledge the requirement of at least two dislocations for endorsement. Therefore, we interviewed a subset of 17 subjects with a positive response on item 4 about their interpretation of the question. Seven reported that they had overlooked this requirement. We do not believe this is a translation error since the question is phrased similarly in both languages. Moreover, we doubt that a more correct interpretation of the stricter meaning actually would change the performance of the 5PQ. Nevertheless, if a revised version of the $5 \mathrm{PQ}$ is to be developed, we propose to phrase this item as "As a child or teenager did your shoulder or kneecap dislocate on at least two occasions? - please note that one occasion doesn't count", and to evaluate whether it improves the performance of the $5 \mathrm{PQ}$.

\section{Limitations}

We do acknowledge that no large scale cultural or intellectual testing was done during the translation process. Although we did not specifically make a cultural validation of the Swedish $5 \mathrm{PQ}$, the performance of our version suggests an acceptable cultural adaption. The low number of individuals with an affirmative response to item 4 "As a child or teenager did your shoulder or kneecap dislocate on more than one occasion?" would have called for a rather extensive pilot group to acknowledge potential misunderstandings regarding this item. The Brazilian-Portuguese version of the 5PQ included illustrations in order to improve the interpretability. In our opinion, however, such addition would result in a revised version of the 5PQ rather than a Swedish translation of the instrument.

\section{Conclusions}

The Swedish translation of the 5PQ demonstrated properties similar to what was reported in other linguistic versions and can be considered a valid and reliable instrument to screen for or identify GJH. Moreover, the recommended cut-off score of $\geq 2$ for $\mathrm{GJH}$ on the $5 \mathrm{PQ}$, was replicated in our study. A revised version of the 5PQ could include illustrations to facilitate its interpretability. Furthermore, a more detailed wording on item 2 and 4 may improve the test performance.

\section{Supplementary information}

Supplementary information accompanies this paper at https://doi.org/10. 1186/s12891-020-3067-1.

Additional file 1: Contains Table S1. The translation process of the Swedish 5PQ.

Additional file 2: Contains Table S2. Evaluation of validity of the Swedish 5PQ. Tested against various cut-off scores on the reference test.

Additional file 3: Contains The Swedish 5PQ Screen print-out version with instructions to the clinician.

\section{Abbreviations}

5PQ: Five-part questionnaire on hypermobility; AUC: Area under the curve; BS: Beighton Score; GJH: Generalised joint hypermobility; ICC: Intra-class correlation coefficient; NPV: Negative predictive value; OR: Odds ratios; PPV: Positive predictive value; ROC: Receiver operator characteristic

\section{Acknowledgements}

We are grateful to Marcus Lithander for providing assistance during data collection.

\section{Authors' contributions}

MG: conception and design, data acquisition, data analysis and interpretation, major contributor in writing the manuscript. $\mathrm{MBH}$ : conception and design, data analysis and interpretation, critical revision of the manuscript. ME: data analysis and interpretation, critical revision of the manuscript. SB: conception and design, data analysis and interpretation, critical revision of the manuscript. All authors read and approved the final manuscript.

\section{Funding}

This work was supported by grants from the Swedish Research Council (K2012- 62X-22130-04-6) to SB. The funders had no role in study design, data collection and analysis, decision to publish, or preparation of the manuscript. Open access funding provided by Örebro University.

\section{Availability of data and materials}

The datasets generated during and/or analysed during the current study are available from the corresponding author on reasonable request.

\section{Ethics approval and consent to participate}

The study was approved by The Regional Ethics Review Board of Stockholm (Approval number. 2014/1742-31 and 2017/1688-31) at Karolinska Hospital. All involvement was voluntary and participants provided written informed consent prior to data collection.

\section{Consent for publication}

Not applicable.

\section{Competing interests}

The authors declare that they have no competing interests.

\section{Author details}

${ }^{1}$ Faculty of Medicine and Health, University Health Care Research Centre, Örebro University, Örebro, Sweden. ${ }^{2}$ School of Medical Sciences, Örebro University, Örebro, SE-70182 Örebro, Sweden. ${ }^{3}$ Department of Clinical Neuroscience, Karolinska Institutet, Stockholm, Sweden.

Received: 20 February 2019 Accepted: 15 January 2020

Published online: 17 March 2020

\section{References}

1. Castori M, Tinkle B, Levy H, Grahame R, Malfait F, Hakim A. A framework for the classification of joint hypermobility and related conditions. Am J Med Genet Part C Semin Med Genet. 2017;175:148-57.

2. Hakim A, Grahame R. Joint hypermobility. Best Pract Res Clin Rheumatol. 2003;17:989-1004

3. Beighton $\mathrm{P}$, Solomon L, Soskolne CL. Articular mobility in an African population. Ann Rheum Dis. 1973;32:413-8. 
4. Hakim AJ, Grahame R. A simple questionnaire to detect hypermobility: an adjunct to the assessment of patients with diffuse musculoskeletal pain. Int J Clin Pract. 2003;57:163-6.

5. Hakim AJ, Cherkas LF, Grahame R, Spector TD, MacGregor AJ. The genetic epidemiology of joint hypermobility: a population study of female twins. Arthritis Rheum. 2004;50:2640-4.

6. De Moraes DA, Baptista CA, Crippa JAS, Louzada-Junior P. Translation into Brazilian Portuguese and validation of the five-part questionnaire for identifying hypermobility. Rev Bras Reumatol. 2011;51:53-69.

7. Buderer NMF. Statistical methodology: I. incorporating the prevalence of disease into the sample size calculation for sensitivity and specificity. Acad Emerg Med. 1996;3:895-900.

8. Malfait F, Francomano C, Byers P, Belmont J, Berglund B, Black J, et al. The 2017 international classification of the Ehlers-Danlos syndromes. Am J Med Genet Part C Semin Med Genet. 2017:175:8-26.

9. The Area Under an ROC Curve n.d. http://gim.unmc.edu/dxtests/roc3.htm (Accessed 13 June 2018).

10. Peat J, Barton B. Medical Statistics : A Guide to SPSS, Data Analysis and Critical Appraisal. 2nd ed: Wiley Blackwell; 2014.

11. Koo TK, Li MY. A guideline of selecting and reporting Intraclass correlation coefficients for reliability research. J Chiropr Med. 2016;15:155-63.

12. Stanhope L, Turnbull K. Introduction to Medical Terminology. 1st ed. USA: The Goodheart-Willcox Company; 2017. https://www.g-wonlinetextbooks. com/introduction-medical-terminology-2017/2.

13. Glans M, Bejerot S, Humble MB. Generalised joint hypermobility and neurodevelopmental traits in a non-clinical adult population. $\mathrm{Br} J$ Psychiatry Open. 2017;3:236-42.

14. Cameron KL, Duffey ML, DeBerardino TM, Stoneman PD, Jones CJ, Owens BD. Association of Generalized Joint Hypermobility with a history of Glenohumeral joint instability. J Athl Train. 2010;45:253-8.

15. Russek LN, Errico DM. Prevalence, injury rate and, symptom frequency in generalized joint laxity and joint hypermobility syndrome in a "healthy" college population. Clin Rheumatol 2016;35:1029-39.

16. Larsson LG, Baum J, Mudholkar GS, Srivastava DK. Hypermobility: prevalence and features in a Swedish population. Br J Rheumatol. 1993:32:116-9.

17. Juul-Kristensen B, Schmedling K, Rombaut L, Lund H, Engelbert RHH. Measurement properties of clinical assessment methods for classifying generalized joint hypermobility-a systematic review. Am J Med Genet Part C Semin Med Genet. 2017:175:116-47.

\section{Publisher's Note}

Springer Nature remains neutral with regard to jurisdictional claims in published maps and institutional affiliations.

Ready to submit your research? Choose BMC and benefit from:

- fast, convenient online submission

- thorough peer review by experienced researchers in your field

- rapid publication on acceptance

- support for research data, including large and complex data types

- gold Open Access which fosters wider collaboration and increased citations

- maximum visibility for your research: over $100 \mathrm{M}$ website views per year

At $\mathrm{BMC}$, research is always in progress.

Learn more biomedcentral.com/submissions 